\title{
Correction to: Mesalazine formulation intolerance due to suspected excipient allergy in the treatment of ulcerative colitis: a case report
}

\author{
Yoshinori Arai ${ }^{1}$ - Maiko Ogawa ${ }^{1} \cdot$ Fumitsugu Yamane ${ }^{1} \cdot$ Natsuki Sumiyoshi $^{1} \cdot$ Rikako Arimoto $^{1} \cdot$ Yoshitaka Ando $^{1}$. \\ Daisuke Endo ${ }^{1} \cdot$ Tatsuya Nakada $^{1} \cdot$ Ichiro Sugawara ${ }^{1} \cdot$ Hiroshi Yokoyama $^{1} \cdot$ Keiko Shimoyama $^{2} \cdot$ Hiroko Inomata $^{2}$. \\ Yosuke Kawahara $^{2} \cdot$ Masayuki Kato $^{2} \cdot$ Seiji Arihiro ${ }^{1} \cdot$ Atsushi Hokari $^{1} \cdot$ Masayuki Saruta $^{3}$
}

Published online: 25 September 2020

(c) Japanese Society of Gastroenterology 2020

\section{Correction to: Clinical Journal of Gastroenterology https://doi.org/10.1007/s12328-020-01216-2}

The article "Mesalazine formulation intolerance due to suspected excipient allergy in the treatment of ulcerative colitis: a case report", written by Yoshinori Arai, Maiko Ogawa, Fumitsugu Yamane, Natsuki Sumiyoshi, Rikako Arimoto, Yoshitaka Ando, Daisuke Endo, Tatsuya Nakada, Ichiro Sugawara, Hiroshi Yokoyama, Keiko Shimoyama, Hiroko Inomata, Yosuke Kawahara, Masayuki Kato, Seiji Arihiro, Atsushi Hokari \& Masayuki Saruta, was originally published electronically on the publisher's internet portal (currently SpringerLink) on 03 September 2020 with open access. With the author(s)' decision to step back from Open Choice, the copyright of the article changed on 21 September 2020 to () Japanese Society of Gastroenterology 2020 and the article is forthwith distributed under the terms of copyright.

The original article has been updated.

The original article can be found online at https://doi.org/10.1007/ s12328-020-01216-2.

Yoshinori Arai

yoshinori.a1@gmail.com

1 Division of Gastroenterology and Hepatology, Department of Internal Medicine, The Jikei University Katsushika Medical Center, -41-2 Aoto, Katsushika-ku, Tokyo 105-8461, Japan

2 Department of Endoscopy, The Jikei University Katsushika Medical Center, Tokyo, Japan

3 Division of Gastroenterology and Hepatology, Department of Internal Medicine, The Jikei University School of Medicine, Tokyo, Japan
Publisher's Note Springer Nature remains neutral with regard to jurisdictional claims in published maps and institutional affiliations. 